\title{
Characterization and cloning of a brittle culm mutant (bc88) in rice (Oryza sativa L.)
}

\author{
RAO YuChun ${ }^{1 \dagger *}$, YANG YaoLong ${ }^{2 \dagger}$, XIN DeDong $^{1}$, LI XiaoJing $^{1}$, ZHAI KaiEn $^{1}$, MA BoJun ${ }^{1}$, \\ PAN JianWei ${ }^{1}$, QIAN Qian ${ }^{2 *} \&$ ZENG DaLi ${ }^{2 *}$ \\ ${ }^{1}$ College of Chemistry and Life Sciences, Zhejiang Normal University, Jinhua 321004, China; \\ ${ }^{2}$ State Key Laboratory of Rice Biology, China National Rice Research Institute, Hangzhou 310006, China
}

Received October 14, 2012; accepted December 14, 2012; published online May 14, 2013

\begin{abstract}
This study characterizes a brittle culm (bc88) mutant of rice (Oryza sativa L.) obtained by ethylene methylsulfonate (EMS)-induced mutagenesis of Wuyunjing 7 . The $b c 88$ mutant exhibits a diversity of pleiotropic phenotypes, including brittle culm at the whole-plant growth stages, withered leaf tips at the seedling stage, and 18-d delay in heading date at the mature stage. Genetic analysis indicates that the $b c 88$ mutant is controlled by a single recessive nuclear gene. The mutated $b c 88$ gene isolated by map-based cloning contains only one point mutation in the 5th exon relative to its wild-type BC88 (LOC_Os09g25490 and Os09g0422500), leading to an amino acid change from $\mathrm{P}$ to $\mathrm{L}$ in $b c 88$ plants. Alignment of the putative protein sequence with its homologs indicates that the mutation is located in the conserved region of the sequence. Detection of the transcription level of $B C 88$ in rice plants shows that the expression level of $B C 88$ is higher in spikes and culms than in leaves, roots, and leaf sheaths. These contribute to understanding of the molecular mechanism of cellulose synthesis. The target gene $B C 88$ can be a useful tool in molecular marker-assisted selection for rice culm trait breeding.
\end{abstract}

rice (Oryza sativa L.), brittle culm, Wuyunjing 7, clone, mutation

Citation: $\quad$ Rao Y C, Yang Y L, Xin D D, et al. Characterization and cloning of a brittle culm mutant (bc88) in rice (Oryza sativa L.). Chin Sci Bull, 2013, 58: 3000-3006, doi: 10.1007/s11434-013-5806-2

Plant cell wall plays an important role in maintaining cell shape and straw stiffness. Most importantly, it serves as a skeletal framework of plants, which affects the lodging resistance of plants and promotes the production of crops [1]. Common components of cell wall include cellulose, hemicelluloses, pectins, lignins, and pectic polysaccharides, of which cellulose is the main constituent of cell wall in most plant species [2,3], and brittleness mutants commonly show variations in cellulose content. Cellulose synthase catalytic subunit (CesA) is an important component that controls the content of cellulose in plants, which indirectly affects the lodging resistance in rice as well as the crop yield.

The CesA, first isolated from cotton [4], commonly contains 8 transmembrane domains with a large cytoplasmic region between the 2 nd and the 3 rd transmembrane domains [5]. So far, 10 CesA genes have been identified in Arabidopsis and 9 in rice and typically classified into 2 groups based on the phylogenetic analysis. Of these, one group regulates the primary cell wall, and the other regulates the secondary cell wall. CesA genes have been cloned in Arabidopsis and rice using molecular genetic methods. Evidence from CesA mutants in Arabidopsis shows that the CesA gene encodes the enzyme responsible for cellulose synthesis [6]. Arabidopsis mutants, i.e., irxl (irregular xylem1), irx3 and irx5 genes destroy the function of AtCesA8, AtCesA7 and AtCesA4, respectively [7-10]. These CesAs form a complex CesA triplex responsible for cellulose synthesis [11]. In rice, OsCesA4, OsCesA7, OsCesA9 form a

$\dagger$ These authors contributed equally to this work.

*Corresponding authors (email: qianqian188@hotmail.com; dalizeng@126.com; ryc@zjnu.cn) 
similar complex that is involved in the synthesis of the secondary cell wall. Once the function of CesA protein is inhibited or lost, individual plants usually become fragile because of the reduction of cellulose content [12-14]. In Arabidopsis, at least 13 irx genes have been identified. Of these, irx6 encodes a member of the COBRA family, which is similar to phytochelatin synthetase and is involved in the biosynthesis of the secondary cell wall [15]. Brittleness mutants are accompanied by a number of chemical changes, such as reduced levels of cellulose and sugars in cell wall, which develop the phenotype of brittle culm. These mutants are of great research value for understanding the mechanism of cellulose formation in plant cell wall.

To date, at least $12 b c$ mutants $(b c 1, b c 2, b c 3, b c 4, b c 5$, $b c 6, b c 7, b c 10, b c 11, b c 12, b c 14$, and $b c 15)$ have been characterized in rice. $B C 1$ shows high similarity to irx 6 in Arabidopsis, regulating the biosynthesis of secondary cell wall to provide the main mechanical strength of rice plants $[16,17]$. $B C 3$ encodes a member of the classical dynamin protein family. It is tightly involved in the synthesis of cellulose and is essential for the formation of the secondary cell wall $[18,19]$. $B C 5$ regulates the formation of secondary cell wall in node sclerenchyma tissues [20]. BC7 and BC11 encode the OsCesA4 protein, which is rich in plasma membrane so that abnormal cell wall is developed [10,13]. Different from $B C 11, B C 12$ is likely regulated by CDKA. It encodes a kinesin- 4 protein and acts as a dual-targeting kinesin protein, regulating the cell-cycle progression [21]. In recent years, some key genes related to cellulose biosynthesis through the brittle culm mutants in rice had been cloned, e.g., BC14 and BC15. BC14 encodes a Golgi nucleotide sugar transporter [22], while $B C 15$ encodes a membraneassociated chitinase-like protein. Both play an important role in polysaccharide synthesis in rice [23]. The previous findings of these genes make a significant contribution to the understanding of the mechanism of cellulose synthesis in plants.

This study characterized a brittle culm 88 mutant of the japonica rice variety Wuyunjing 7 obtained by ethylene methylsulfonate (EMS)-induced chemical mutagenesis. Genetic analysis indicated that $b c 88$ was controlled by a single recessive nuclear gene. The mutation site of $b c 88$ was identified by map-based cloning, which caused various morphological and physiological changes. $B C 88$ is related to cellulose synthesis, which is allelic to $B C 6$.

\section{Materials and methods}

\subsection{Plant materials}

The mutant was obtained from mutagenesis of the Wuyunjing 7 variety induced by EMS (Sigma Corporation, USA). In this paper, the plant with brittle culm is referred to as the $b c 88$ mutant. Crosses were made between $b c 88$ and conventional varieties Nipponbare, TN1, ZF802, and NJ06.
EMS-treated seeds from the $\mathrm{F}_{1}$ and $\mathrm{F}_{2}$ generations were used for further researches. The $\mathrm{F}_{2}$ individuals with the mutant phenotype were selected for gene mapping. The rice plants, including $b c 88$, conventional rice varieties, and $\mathrm{F}_{1}$ and $\mathrm{F}_{2}$ generations were transplanted individually in the field in Hangzhou $\left(120^{\circ} 00^{\prime} \mathrm{E}, 30^{\circ} 06^{\prime} \mathrm{N}\right)$ in spring, or in the Linshui County $\left(110^{\circ} 02^{\prime} \mathrm{E}, 18^{\circ} 03^{\prime} \mathrm{N}\right)$ in winter.

\subsection{Genetic analysis of mutant $b c 88$}

As $b c 88, b c 1$ [16], $b c 3$ [19], $b c 10$ [24], $b c 11$ [13] and $b c 12$ [21] share similar phenotypes, crosses between $b c 88$ and the other 5 mutants $(b c 1, b c 3, b c 10, b c 11$ and $b c 12)$ were made. The phenotypes of the $F_{1}$ and $F_{2}$ generations of different crosses were recorded. The separation ratios were calculated to determine whether these genes were allelic. In addition, reciprocal crosses between $b c 88$ and 4 common varieties (Nip, TN1, ZF802, and NJ06) were made to study the genetic mechanism of $b c 88$. The software SAS 8.0 was used to compute the Chi-square value.

\subsection{Identification of linkage marker}

Total DNA was extracted from fresh rice leaves using the CTAB method as described by Sun et al. [25] with slight modifications. Twenty individual plants with brittle culm phenotype were selected from the populations of " $b c 88$ / TN1" and " $b c 88 / \mathrm{ZF} 802 "$, respectively. The bulked segregant analysis (BSA) [26] was used to identify candidate markers linked to $b c 88$.

\subsection{Gene mapping of $\mathrm{BC} 88$}

Polymorphism analyses of the parents of the segregation population were accomplished using simple sequence repeat (SSR) markers. The $\mathrm{F}_{2}$ generation was used to screen polymorphic markers to analyze linkage groups of the $b c 88$ gene, and the linked markers were carried through to linkage analysis of the mutant individuals. The simple sequence repeat (SSR) markers were uniformly distributed over the 12 rice chromosomes. The PCR assay was carried out according to Panaud et al. [27] using an EDC-810 PCR machine (DongSheng Biotech Corporation). The PCR products were checked by running $4 \%$ agarose gel electrophoresis. After ethidium bromide staining, the gel was virtualized and observed using a gel imaging system (ALphalmager EP, Alpha Innotech Corporation, USA). Primers were designed for the sequence-tagged site markers and cleaved amplified polymorphic sequence markers using Primer Premier 5 according to the differences in sequence between japonica (http://rgp.dna.affrc.go.jp) and indica var. 93-11 (http:// www.rise.genomics.org.cn) on chromosome 9. The designed primers were used for fine mapping of the $B C 88$ gene. 


\subsection{Identification of candidate genes}

To identify the candidate genes, the genomic sequence containing $B C 88$ was examined using the websites for Nipponbare genome prediction and full-length cDNA sequences (http://rice.plantbiology.msu.edu/cgi-bin/gbrowse/rice/).

The alleles of the candidate genes for the two mapping parents as well as its wild-type were sequenced and then compared with each other to detect potential differences. Primers were designed for sequencing using the Primer Premier 5 software. Multiple sequence alignment was accomplished using program CLUSTALX 2.0.

\subsection{Detection of $\mathrm{BC} 88$ expression}

At the heading stage, total RNA was extracted from the root, stem, sheath, leaf, and spike of $b c 88$ using the TRIzol reagent (Invitrogen, USA). The extracted RNA was converted into first-strand cDNA using the Rever Tra qPCR RT kit (Toyobo Corporation, China). BC88 gene expression was studied by real-time quantitative PCR (Biometra) using $B C 88$ gene full-length cDNA as template.

\section{Results}

\subsection{Morphological characteristics of $b c 88$}

The $b c 88$ was brittle at the whole-plant growth stages, with culms easily broken by bending (Figure 1(b)). Withered leaf tips appeared in $b c 88$ from the seeding stage to the mature stage (Figure 1(c)), which facilitated sampling. The average tiller number of $b c 88$ was 6 , much less than the wild-type
Wuyunjing 7 at mature stage (Figure 1(a), Table 1). Comparing to the wild-type, $b c 88$ showed an 18-d delay in heading date. All spikelets in the wild-type were heading completely, while only some spikelets emerged in panicles of $b c 88$ (Figure 1(a)). The stems of $b c 88$ were approximately $60 \mathrm{~cm}$ in height, much shorter than those of the wild-type (Figure 1(a), Table 1). Comparison of the vegetative shoots length between $b c 88$ and its wild-type showed that each shoot of the former was shorter than that of the latter (Figure $1(\mathrm{~d})$ ). In addition, $b c 88$ was accompanied by a number of pleiotropic phenotypes, e.g., shorter roots (Figure 1(e)), and fewer numbers of secondary branches (Table 1). However, $b c 88$ and its wild-type were not apparently different in some agronomic traits, including the percentage of seed-setting and the number of primary branches (Table 1). In addition, the cellulose content was reduced by approximately $40 \%$ in the fresh weight of $b c 88$, accounting for the bittle phenotype of $b c 88$ (Table 1).

\subsection{Genetic analysis of mutant $b c 88$}

The $b c 88$ consistently exhibited the mutant phenotype after several generations of continuous selfing. Crosses between $b c 88$ and other mutants with similar phenotypes $(b c 1, b c 3$, $b c 10, b c 11$ and $b c 12$ ) suggest that $b c 88$ is not allelic with any of those genes. The $\mathrm{F}_{1}$ population exhibited a wild-type phenotype, with no brittle phenotype observed in $\mathrm{F}_{1}$ population, similar to the wild-type. In the $\mathrm{F}_{2}$ generation, all of the $P$-values were $>0.05$, and the ratio of normal phenotype to $b c 88$ in each combination was close to 9:7 (Table 2), corresponding to the genetic relationships of two non-allelic
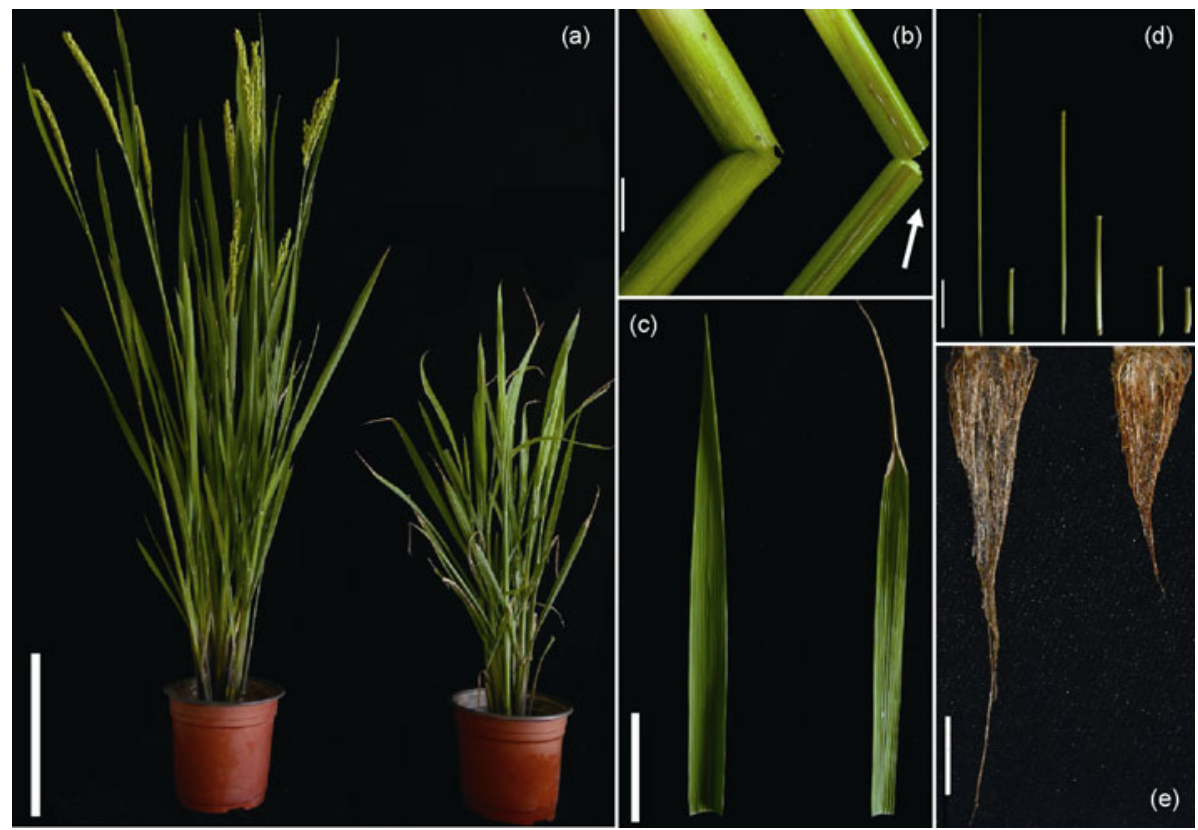

Figure 1 The morphological differences between $b c 88$ and its wild-type plants. (a) Left, wild-type; right, $b c 88$; bar= $25 \mathrm{~cm}$. (b) Left, a culm of wild-type; right, an easily broken $b c 88$ culm (arrow); bar $=0.75 \mathrm{~cm}$. (c) $b c 88$ leaf tip phenotype. Left, wild-type; right, $b c 88$; bar $=2 \mathrm{~cm}$. (d) Comparison of the internode length of the $b c 88$ mutant compared to its wild-type. Left, wild-type; right, $b c 88$; bar=4 cm. (e) Root length of the $b c 88$ mutant compared to its wild-type. Left, wild-type; right, $b c 88$; bar $=4 \mathrm{~cm}$. 
Table 1 Comparison of the major agronomic traits between $b c 88$ and its wild-type rice

\begin{tabular}{|c|c|c|c|c|c|c|}
\hline Agronomic traits & Height $(\mathrm{cm})$ & Tiller number & $\begin{array}{c}\text { Percentage of } \\
\text { seed-setting }(\%)\end{array}$ & $\begin{array}{c}\text { Number of } \\
\text { primary branches }\end{array}$ & $\begin{array}{c}\text { Number of } \\
\text { secondary branches }\end{array}$ & $\begin{array}{c}\text { Cellulose content } \\
\left(\mathrm{mg} \mathrm{g}^{-1} \text { fresh weight }\right)\end{array}$ \\
\hline Wild-type & $91.5 \pm 5.25$ & $9.2 \pm 1.05$ & $87.90 \pm 9.83$ & $14.67 \pm 2.41$ & $39.67 \pm 7.25$ & $61.8 \pm 3.43$ \\
\hline$b c 88$ & $60.4 \pm 4.35$ & $6.1 \pm 1.24$ & $85.42 \pm 10.15$ & $13.3 \pm 3.26$ & $15.67 \pm 3.35$ & $37.4 \pm 2.15$ \\
\hline
\end{tabular}

Table 2 Genetic analysis of $\mathrm{F}_{2}$ populations derived from crosses between $b c 88$ and other similar mutants

\begin{tabular}{|c|c|c|c|c|c|c|}
\hline \multirow{2}{*}{ Combination } & \multicolumn{2}{|c|}{$\mathrm{F}_{1}$} & \multicolumn{2}{|c|}{$\mathrm{F}_{2}$} & \multirow{2}{*}{$\chi^{2}(9: 7)$} & \multirow{2}{*}{$P$-value } \\
\hline & Wild-type & $b c 88$ & Wild-type & $b c 88$ & & \\
\hline$b c 88 / b c 1$ & 7 & 0 & 123 & 90 & 0.1938 & 0.6597 \\
\hline$b c 88 / b c 10$ & 6 & 0 & 108 & 75 & 0.5691 & 0.4506 \\
\hline$b c 88 / b c 11$ & 5 & 0 & 98 & 71 & 0.2075 & 0.6488 \\
\hline$b c 88 / b c 12$ & 10 & 0 & 203 & 152 & 0.1256 & 0.7230 \\
\hline
\end{tabular}

genes. The $F_{1}$ populations derived from the reciprocal crosses between $b c 88$ and other common varieties (Nip, TN1, ZF802, and NJ06) showed normal phenotypes. These results indicate that $b c 88$ is controlled by a recessive nuclear gene (Table 3). Further analysis of the phenotypes in the $\mathrm{F}_{2}$ generation and the calculation of Chi-square value showed that the ratio of wild-type phenotype to $b c 88$ in each combination was close to $3: 1$. This confirms that the characteristic of $b c 88$ is qualitative and controlled by a single recessive nuclear gene.

\subsection{Fine mapping and cloning of $b c 88$}

A total of 108 pairs of SSR markers, which were polymorphic between the parents and uniformly distributed over the 12 rice chromosomes with intervals ranging from $10-20 \mathrm{cM}$, were selected for detection of amplification diversity between $F_{1}$ genomic DNA and mutant pool DNA as well as for primary mapping of the target gene. The marker RM566, located on chromosome 9, exhibited significant amplification diversity between $F_{1}$ genomic DNA and mutant pool DNA. Twenty individual plants that were used to construct the mutant pool were tested whether RM566 was linked with the target gene phenotype and the result was positive.
New markers close to RM566 were developed for PCR amplification and detection. The results show that the molecular marker RM3700 is linked with the target gene and its recombinant individuals are different from those of RM566. This indicates that the $B C 88$ gene is located between the markers RM566 and RM3700 (Figure 2(a)). Among the 1082 mutant individuals separated by RM566 and RM3700, 45 and 11 recombinant individuals were detected, based on which the genetic distances between the BC88 gene and RM566 or RM3700 were calculated, i.e., 2 and $0.5 \mathrm{cM}$, respectively. For fine mapping, markers were developed between RM566 and RM3700 (Table 4). Then, the candidate region containing the $B C 88$ locus was narrowed between the markers P2 and P9, containing 4 BACs (Figure 2(b)). The STS markers P2, P3, P4, P5 and P6 revealed 13, 8, 7, 4 and 1 recombinants, respectively (Figure 2(c)), while the markers $\mathrm{P} 9, \mathrm{P} 8$ and $\mathrm{P} 7$ indicated 7, 3 and 1 recombinants, respectively. The $B C 88$ gene is located in a $65.4-\mathrm{kb}$ DNA region between the markers P6 and P7 (Figure 2(c)), which contains 9 ORFs. However, sequence comparison involving the region from the mapping parents and Wuyunjing 7 shows that only one of these candidate genes (LOC_ Os09g25490) is polymorphic (Figure 3). bc88 has one base-pair substitution only at position 1784 in the 5 th exon

Table 3 Genetic analysis of $\mathrm{F}_{2}$ populations derived from crosses between $b c 88$ and other common varieties

\begin{tabular}{|c|c|c|c|c|c|c|}
\hline \multirow{2}{*}{ Combination } & \multicolumn{2}{|c|}{$\mathrm{F}_{1}$} & \multicolumn{2}{|c|}{$\mathrm{F}_{2}$} & \multirow{2}{*}{$\chi^{2}(3: 1)$} & \multirow{2}{*}{$P$-value } \\
\hline & Wild-type & $b c 88$ & Wild-type & $b c 88$ & & \\
\hline bc $88 / \mathrm{Nip}$ & 9 & 0 & 208 & 63 & 0.444 & 0.5052 \\
\hline$b c 88 / \mathrm{TN} 1$ & 7 & 0 & 184 & 54 & 0.6779 & 0.4103 \\
\hline $\mathrm{TN} 1 / b c 88$ & 9 & 0 & 221 & 65 & 0.7879 & 0.3747 \\
\hline$b c 88 / \mathrm{ZF} 802$ & 11 & 0 & 254 & 80 & 0.1956 & 0.6583 \\
\hline ZF802/bc88 & 12 & 0 & 265 & 84 & 0.1614 & 0.6879 \\
\hline$b c 88 / \mathrm{NJ} 06$ & 11 & 0 & 243 & 76 & 0.2351 & 0.6278 \\
\hline NJ06/bc88 & 14 & 0 & 288 & 89 & 0.3899 & 0.5323 \\
\hline
\end{tabular}


Table 4 Markers for BC88 gene mapping

\begin{tabular}{lll}
\hline Primer & Forward primer $\left(5^{\prime} \rightarrow 3^{\prime}\right)$ & Reverse primer $\left(5^{\prime} \rightarrow 3^{\prime}\right)$ \\
\hline RM566(P1) & ACCCAACTACGATCAGCTCG & CTCCAGGAACACGCTCTTTC \\
P2 & GAGGCGGTCAGGTGGATGCT & CGCCAAACGGGCAAGCAA \\
P3 & GGAGTCCCATCCAAACATAGT & GGTGGCAGAGCAGGTTGTC \\
P4 & CGGTGGATCTTCGTCTTG & TCTGGTGGTAGATTGGTAGTG \\
P5 & TAGTGGCCCAATCTCCTT & CAGTGCCCTGGTTCTTCT \\
P6 & CTGCGTTGGTTAGGAGTC & ATGCGTGAGCAGAAGTCC \\
P7 & TATCTCCAGTTCTCCACCCAC & GGAAGCGGAGGAATTATTGA \\
P8 & GACAGGGAGTTCTGGATC & CACATATCACAGGCTACA \\
P9 & CTGACCGTCCGATCTAACATG & TCGCCGTGGAATGAGTTT \\
RM3700(P10 $)$ & AAATGCCCCATGCACAAC & TTGTCAGATTGTCACCAGGG \\
\hline
\end{tabular}

(a)

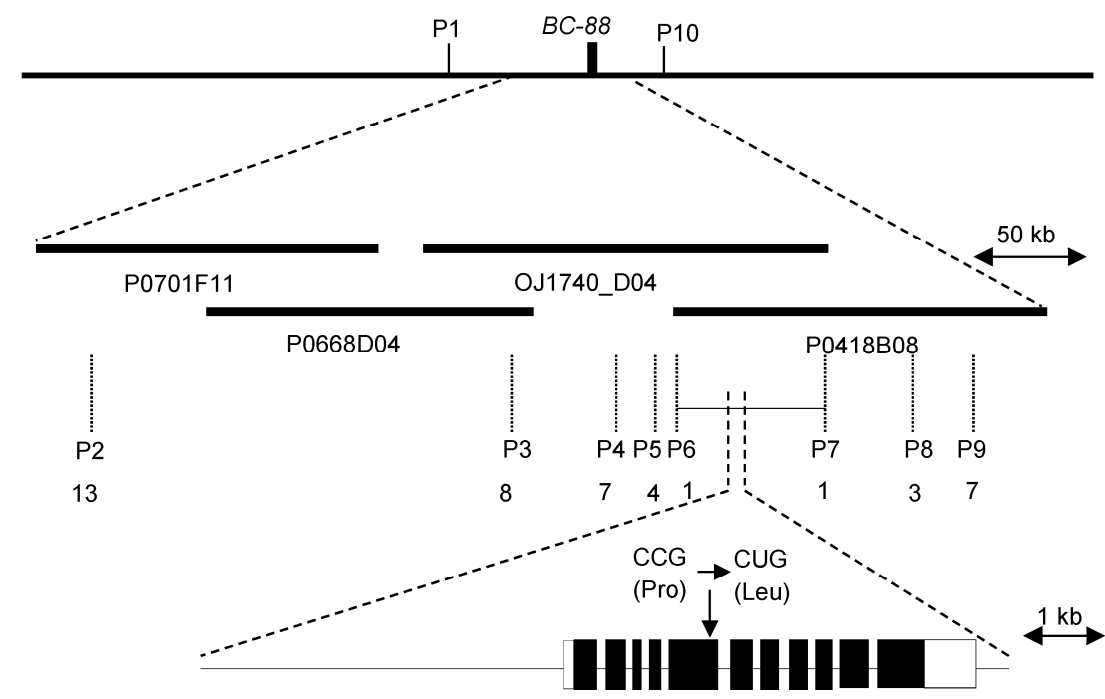

Figure 2 Fine mapping of $B C 88$. (a) $B C 88$ is located between the markers RM566 and RM3700. (b) $B C 88$ is mapped to the region between the markers P2 and P9, consisting of 4 BACs. (c) $B C 88$ is mapped to a $65.4-\mathrm{kb}$ region between the markers P6 and P7. (d) $B C 88$ structure and the mutation site in $b c 88$. The white boxes represent the $5^{\prime}$ and $3^{\prime}$ untranslated regions, the black boxes represent the coding sequences and lines between boxes represent introns. The asterisk indicates the mutation site.

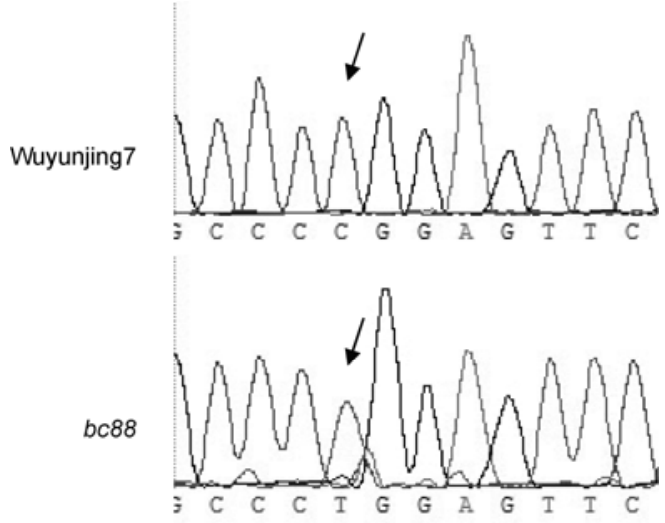

Figure 3 Sequencing of Wuyunjing 7 and $b c 88$, mutation site (arrow).

of BC88 (LOC_Os09g25490, Os09g0422500) compared to the Wuyunjing 7 variety of rice. This missense mutation, changed from CCG to CTG, leads to the variation in 421th amino acid (proline to leucine) in $b c 88$ plants (Figure 2(d)).

\subsection{Expression analysis of $\mathrm{BC} 88$}

Tissue-specific expression of the $B C 88$ gene at the heading stage revealed that $B C 88$ was expressed universally in rice. The expression level of $B C 88$ was found significantly higher in spikes and culms than in root, leaf and leaf sheath (Figure 4).

\section{Discussion}

A series of mutants for various traits in rice form the basis of genetic analysis and functional genomics [28-30]. There are a large number of $B C$ genes identified in rice and Arabidopsis [31]. In addition to brittle phenotype, this study found substantial changes between $b c 88$ and its wild-type. The most significant difference between $b c 88$ and other 


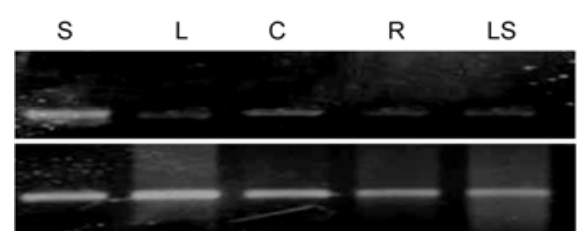

$B C 88$

Actin 1

Figure 4 Expression of $B C 88$ in various tissues at the heading stage. S, spike; L, leaf; C, culm; R, root; and LS, leaf sheath; Rice Actin1 used as control. BC88 Primer for $B C 88$ : F, ACCCACCCAAGGGACCCAAGAG; R, ACAAACTCGCAAACGTGCTAATCG.

brittle rice mutants is related to the 18-d delay in heading date in $b c 88$. That is, when all spikelets of the wild-type were heading completely, only part of the spikelets emerged in panicles of $b c 88$ (Figure 1(a)). However, the seed setting rate of $b c 88$ was almost the same as that of its wild-type, possibly due to the impact of $B C 88$ on the expression of flowering genes. In addition, the $b c 88$ exhibited a semidwarf phenotype compared with the wild-type. Subsequent measurement of each intermodal elongation of $b c 88$ as well as its wild-type showed that the former was shorter than the latter. Thus, $b c 88$ belongs to the dn type of dwarf mutants (Figure 1(d))[32].

More recently, an allelic to $B C 88$, named $B C 6$ has been reported [5]. Although $b c 88$ and $b c 6$ were both obtained by EMS treatment, their wild-type was different, i.e., Wuyunjing 7 variety for the former and IR68 variety for the latter. The phenotype of $b c 88$ is recessive, different from the semi-dominant brittle phenotype of $b c 6$. In addition, $b c 88$ shows pleiotropic phenotypes similar to $b c 3$ and $b c 11 \mathrm{mu}-$ tants, such as dwarfism or withered leaf tips $[19,13,33]$. In contrast, no pleiotropic phenotypes have been observed in $b c 6$. $b c 88$ shows only one point mutation in the 5 th exon of Os09g0422500, while the mutation incident occurred in the 7 th exon of Os09g0422500 in bc6. Both of them have missense mutation. Regarding the more severe phenotype of $b c 88$ compared to $b c 6$, we speculate that the mutation of bc 88 occurs in a conserved region that is critical to protein function, resulting in the more significant inhibitory effect than $b c 6$.

DNA sequencing demonstrates that $B C 88$ gene has one nucleotide substitution, whereas the allelic sequence of $b c 88$ is exactly the same as that of Wuyunjing 7. According to the Rice Genome Annotation website (http://rice.plantbiology. msu.edu/), the candidate gene for $b c 88$ encodes a cellulose synthase. Thus, $B C 88$ is related to the cellulose synthesis pathway, consistent with the finding in BC6 [5]. Cellulose is a major component of plant cell wall, which can be grouped into three basic types according to the wall thickness, i.e., parenchyma, collenchyma, and sclerenchma [33]. Toshihisai has indicated that $B C 6$ is a secondary cell wall-specific CESA. Hence, $B C 88$ likely plays an important role in the secondary cell wall as well. Multiple sequence alignment of BC88 protein with its homologs in rice and other species shows that the mutation occurs in a highly conserved region
(Figure 5), thus may exert a significant negative effect on the protein function. Tansmembrane structure analysis indicates that the $\mathrm{BC} 88$ protein consists of 8 transmembrane domains. This suggests that $B C 88$ encodes a membrane protein (Figure 6). RT-PCR assay of $B C 88$ expression in different tissues shows that the expression reached much higher levels in spike and culm than in leaf, root, and leaf sheath. Further functional analysis of $B C 88$ remains difficult due to the low transgene efficiency of the Wuyunjing 7 variety as well as the $b c 88$ mutant.

Plant mechanical strength is an important agronomic trait in rice. To enhance the mechanical strength in rice improves the lodging resistance in rice plants while reducing the loss of crop yield. On the other side, rice varieties with brittle culm could reduce the energy consumption of straw processing, producing materials easily serving as animal feed and straw returning. The change in mechanical strength of rice stem, usually accompanied with the change in cell wall structure or composition, is contributed to the transformation from straw ingredients to efficient biomass by manual adjustment. The cloning of $B C 88$ is beneficial to the understanding of the regulation mechanism of plant mechanical strength, and the breeding of lodging-resistant varieties or plants with brittle culms.
(a) $b c 88$

Z. mays
H. vulgare
A. thaliana
P. taeda
S. moellendorffii
P. patens

(b) $\mathrm{BC} 88$ Os03g0837100 Os07g0424400 Os07g0252400 Os03g0808100 Os07g0208500 Os05g0176100
1

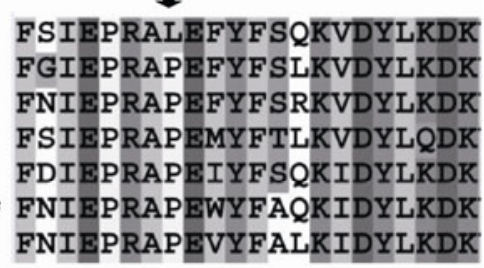

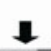

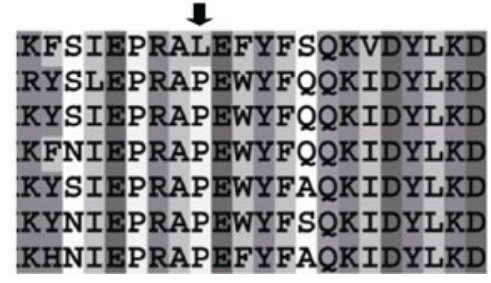

Figure 5 Alignment of amino acid sequences of $B C 88$ with other similar sequences. (a) Among different species; and (b) in Oryza sativa L. The arrow indicates the residue mutated in $b c 88$. Accession numbers for the sequences of OsCesAs and relevant sequence in other species are as follows: NP_001105532.1; BAJ85783.1; NP_197244.1; AAX18649.1; XP_002963550.1; XP_001767133.1; Os03g0837100; Os07g0424400; Os07g0252400; Os03g0808100; Os07g0208500; Os05g0176100.

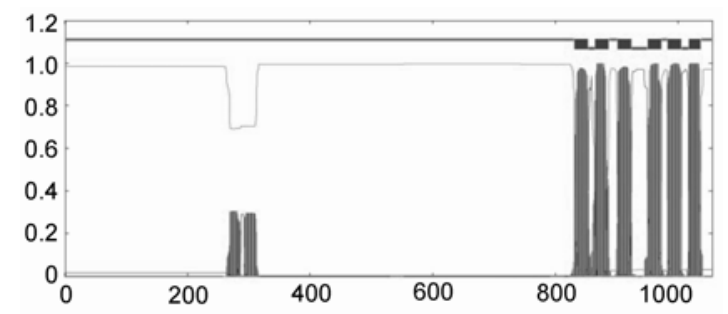

Figure 6 Transmembrane structure analysis of BC88 protein. 
This work was supported by the National Natural Science Foundation of China (30971760, 31201183), the Zhejiang Provincial Qianjiang Talents Program of China (2010R10085), and the Zhejiang Program of Education Department (Y201225687).

1 Hu B L, Kong X L, Bao J S, et al. Advances of studies on gene localization and cloning for plant cell wall traits (in Chinese). Acta Agricult Jiangxi, 2006, 18: 17-21

2 Bacic A, Harris P J, Stone B A. Structure and function of plant cell walls. In: Preiss J, ed. The Biochemistry of Plants. A Comprehensive Treatise. New York: Academic Press, 1988. 14: 297-372

3 Carpita N, McCann M. The cell wall. In: Croteau R, Kutchan T M, Lewis N G, eds. Biochemistry and Molecular Biology of Plants. Rockville: American Society of Plant Physiologists, 2000. 52-108

4 Pear J R, Kawagoe Y, Schreckengost W E, et al. Higher plants contain homologs of the bacterial celA genes encoding the catalytic subunit of cellulose synthase. Proc Natl Acad Sci USA, 1996, 93: 12637-12642

5 Kotake T, Aohara T, Hirano K, et al. Rice Brittle culm 6 encodes a dominant-negative form of CesA protein that perturbs cellulose synthesis in secondary cell walls. J Exp Bot, 2011, 62: 2053-2062

6 Arioli T, Peng L, Betzner A S, et al. Molecular analysis of cellulose biosynthesis in Arabidopsis. Science, 1998, 279: 717-720

7 Taylor N G, Scheible W R, Cutler S, et al. The irregular xylem3 locus of Arabidopsis encodes a cellulose synthase required for secondary cell wall synthesis. Plant Cell, 1999, 11: 769-779

8 Turner S R, Somerville C R. Collapsed xylem phenotype of Arabidopsis identifies mutants deficient in cellulose deposition in the secondary cell wall. Plant Cell, 1997, 9: 689-701

9 Taylor N G, Laurie S, Turner S R. Multiple cellulose synthase catalytic subunits are required for cellulose synthesis in Arabidopsis. Plant Cell, 2000, 12: 2529-2539

10 Taylor N G, Howells R M, Huttly A K, et al. Interactions among three distinct CesA proteins essential for cellulose synthesis. Proc Natl Acad Sci USA, 2003, 100: 1450-1455

11 Haigler $\mathrm{C} \mathrm{H}$, Brown R M. Transport of rosettes from the Golgi apparatus to the plasma membrane in isolated mesophyll cells of Zinnia elegans during differentiation to tracheary elements in suspension culture. Protoplasma, 1986, 134: 111-120

12 Tanaka K, Murata K, Yamazaki M, et al. Three distinct rice cellulose synthase catalytic subunit genes required for cellulose synthesis in the secondary wall. Plant Physiol, 2003, 133: 73-83

13 Zhang B C, Deng L W, Qian Q, et al. A missense mutation in the transmembrane domain of CESA4 affects protein abundance in the plasma membrane and results in abnormal cell wall biosynthesis in rice. Plant Mol Biol, 2009, 71: 509-524

14 Yan C J, Yan S, Zeng X H, et al. Fine mapping and isolation of $B c 7(t)$, allelic to OsCesA4. J Genet Genomics, 2007, 34: 1019-1027

15 Brown D M, Zeef L A, Ellis J, et al. Identification of novel genes in Arabidopsis involved in secondary cell wall formation using expression profiling and reverse genetics. Plant Cell, 2005, 17: 2281-2295

16 Li Y H, Qian Q, Zhou Y H, et al. BRITTLE CULM1, which encodes a COBRA-like protein, affects the mechanical properties of rice plants.
Plant Cell, 2003, 15: 2020-2031

17 Ching A, Dhugga K S, Appenzeller L, et al. Brittle stalk 2 encodes a putative glycosylphosphatidylinositol-anchored protein that affects mechanical strength of maize tissues by altering the composition and structure of secondary cell walls. Planta, 2006, 224: 1174-1184

18 Xiong G Y, Li R, Qian Q, et al. The rice dynamin-related protein DRP2B mediates membrane trafficking, and thereby plays a critical role in secondary cell wall cellulose biosynthesis. Plant J, 2010, 64: $56-70$

19 Hirano K, Kotake T, Kamihara K, et al. Rice BRITTLE CULM 3 $(B C 3)$ encodes a classical dynamin OsDRP2B essential for proper secondary cell wall synthesis. Planta, 2010, 232: 95-108

20 Aohara T, Kotake T, Kaneko Y, et al. Rice BRITTLE CULM 5 (BRITTLE NODE) is involved in secondary cell wall formation in the sclerenchyma tissue of nodes. Plant Cell Physiol, 2009, 50: 1886-1897

21 Zhang M, Zhang B C, Qian Q, et al. Brittle Culm 12, a dual-targeting kinesin-4 protein, controls cell-cycle progression and wall properties in rice. Plant J, 2010, 63: 312-328

22 Zhang B C, Liu X L, Qian Q, et al. Golgi nucleotide sugar transporter modulates cell wall biosynthesis and plant growth in rice. Proc Natl Acad Sci USA, 2011, 108: 5110-5115

23 Wu B, Zhang B C, Dai Y, et al. Brittle Culm15 encodes a membrane-associated chitinase-like protein required for cellulose biosynthesis in rice. Plant Physiol, 2012, 159: 1440-1452

24 Zhou Y H, Li S B, Qian Q, et al. BC10, a DUF266-containing and Golgi-located type II membrane protein, is required for cell-wall biosynthesis in rice (Oryza sativa L.). Plant J, 2009, 57: 446-462

25 Sun C, Chen G, Rao Y C, et al. A simple method for rapid preparation of genomic DNA (in Chinese). Chin J Rice Sci, 2010, 24: 677-680

26 Michelmore R W, Paran I, Kesseli R V. Identification of markers linked to disease-resistance genes by bulked segregant analysis: A rapid method to detect markers in specific genomic regions by using segregating populations. Proc Natl Acad Sci USA, 1991, 88: 98289832

27 Panaud O, Chen X, McCouch S R. Frequency of microsatellite sequences in rice (Oryza sativa L.). Genome, 1995, 38: 1170-1176

28 Zeng D L, Yan M X, Wang Y H, et al. Dul, encoding a novel Prp1 protein, regulates starch biosynthesis through affecting the splicing of $W x^{b}$ pre-mRNAs in rice (Oryza sativa L.). Plant Mol Biol, 2007, 65: 501-509

29 Lin H, Wang R X, Qian Q, et al. DWARF27, an iron-containing protein required for the biosynthesis of strigolactones, regulates rice tiller bud outgrowth. Plant Cell, 2009, 21: 1512-1525

30 Li H G, Xue D W, Gao Z Y, et al. A putative lipase gene EXTRA GLUME1 regulates both empty-glume fate and spikelet development in rice. Plant J, 2009, 57: 593-605

31 Zhang B C, Zhou Y H. Rice Brittleness Mutants: A way to open the 'black box' of monocot cell wall biosynthesis. J Integr Plant Biol, 2011, 53: 136-142

32 Takeda K. Internode elongation and dwarfism in some gramineous plants. Gamma Field Sym, 1977, 16: 1-18

33 Xu J D, Zhang Q F, Zhang T, et al. Phenotypic characterization, genetic analysis and gene-mapping for a brittle mutant in rice. J Integr Plant Biol, 2008, 50: 319-328

Open Access This article is distributed under the terms of the Creative Commons Attribution License which permits any use, distribution, and reproduction in any medium, provided the original author(s) and source are credited. 